\title{
Influence de la couche de nucléation sur le comportement thermomécanique du HEMT
}

\section{Influence of the nucleation layer on the thermomechanical behavior of HEMT}

\author{
Abdelhamid Amar ${ }^{1,2^{\star}}$, Bouchaïb Radi ${ }^{1}$, Abdelkhalak El Hami ${ }^{2}$ \\ ${ }^{1}$ Hassan First University of Settat, FST, LIMII, Route de Casa, Settat 26000, Morocco; bouchaib.radi@yahoo.fr \\ ${ }^{2}$ LMN, INSA Rouen, Normandie University, 76000 Rouen, France; abdelkhalak.elhami@insa-rouen.fr \\ abdelhamid.amar@insa-rouen.fr
}

\begin{abstract}
RÉSUMÉ. Le but principal de cet article, c'est d'étudier l'influence des paramètres géométriques de la structure du transistor à haute mobilité d'électron (HEMT). Nous allons développer la modélisation électro-thermomécanique par la méthode des éléments finis, à l'aide de logiciel Comsol multiphysics. Ce modèle nous permis de simuler le comportement thermomécanique du HEMT en fonction des conditions du fonctionnement. II nous permit également d'étudier l'influence de la couche de nucléation sur ce comportement. Les résultats des simulations numériques obtenues ont montré que malgré que l'épaisseur de la couche ne dépasse pas $1 \mu \mathrm{m}$, elle présente une grande influence sur le comportement thermique et mécanique du composent. Par conséquent, cette couche doit être prise en considération pour toute étude qui vise de développer ou d'optimiser cette technologie.

ABSTRACT. The main goal of this paper is to study the influence of geometrical parameters of the high electron mobility transistor (HEMT) structure. We will develop the electro-thermomechanical modeling by the finite element method, using Comsol multiphysics software. This model allowed us to simulate the thermomechanical behavior of the HEMT according to the operating conditions. It also allowed us to study the influence of the nucleation layer on this behavior. The results of the numerical simulations obtained showed that, although the thickness of the layer does not exceed $1 \mu \mathrm{m}$, it has a great influence on the thermal and mechanical behavior of the component. Therefore, this layer must be taken into consideration for any study that aims to develop or optimize this technology.

MOTS-CLÉS. Modélisation thermomécanique, FEM, HEMT, couche de nucléation.

KEYWORDS. Thermomechanical modeling, FEM, HEMT, nucleation layer.
\end{abstract}

\section{Introduction}

Le transistor à haute mobilité d'électron (HEMT) à base de nitrure d'aluminium-gallium/nitrure gallium $(\mathrm{AlGaN} / \mathrm{GaN})$, est un composant électronique très important da,s les systemes à haute puissance, grâce à sa structure et ses matériaux. Le nitrure de gallium $\mathrm{GaN}$ se caractérise par une grande mobilité, un champ de claquage électrique très élevé et une grande conductivité thermique [SAJ 20]. Grâce à ces caractéristiques et autres, ces transistors ont été largement utilisés dans plusieurs applications à haute fréquence et à haute température [AMA 21b][GAR 16][WAN 19], tels que, la télécommunication, la guerre électronique (domaine militaire) et les systèmes aéroportés [JEO 18][ DON 19]. Le HEMT a été utilisé aussi dans plusieurs systèmes tels que les amplificateurs à haute puissance, les radars et les satellites [KAO 20][CHE 19].

Plusieurs travaux ont traité la modélisation électrique, thermique ou électrothermique, pour simuler le comportement électrique et thermique du transistor en fonction des paramètres électriques et des conditions du fonctionnement [LEN 15 ][AMA 20a][AMA 20b].Dans ce travail on propose une autre méthode multiphysique de modélisation, qui permet de simuler le comportement électrothermomécanique du HEMT. Et d'étudier également l'influence des paramètres géométrique sur ce comportement, comme la couche de la nucléation dans notre cas d'étude. La modélisation sera faite par la méthode des éléments finis en utilisant le logiciel Comsol multiphysics [AMA 19][COM 20]. 
Cet article est composé de plusieurs parties, on va commencer par une description de la technologie HEMT et sa structure, afin d'avoir une idée sur sa topologie ainsi les propriétés thermiques et physiques de ses matériaux. Par la suite on va développer un modèle éléments finis avec le logiciel Comsol multiphysics dans sa partie thermomécanique, qui permettra de manipuler les différents paramètres : la géométrie, les propriétés physiques et les charges etc. Après on développe le modèle éléments finis qui permet d'étudier l'influence de quelques paramètres telles que la puissance dissipée et la couche de nucléation sur le comportement thermomécanique du HEMT.

\section{Description du composant.}

La technologie HEMT est caractérisée par une structure particulière qui se compose de plusieurs couches de différents matériaux. Une couche en carbure de silicium qui représente le substrat sur lequel on construit le composant, la couche de GaN qui contient le 2DEG dans sa partie supérieure, la couche $\mathrm{AlGaN}$ sert à créer une hétérojonction avec la couche de GaN [NIF 17] [AMA 21b]. La couche de nucléation ou la résistance thermique à l'interface sert à réaliser un accord de maillage pour la couche de $\mathrm{SiC}$ et de $\mathrm{GaN}$, une autre fonction est de réduire les contraintes mécaniques et les défauts dans la couche de GaN [AMA 21].

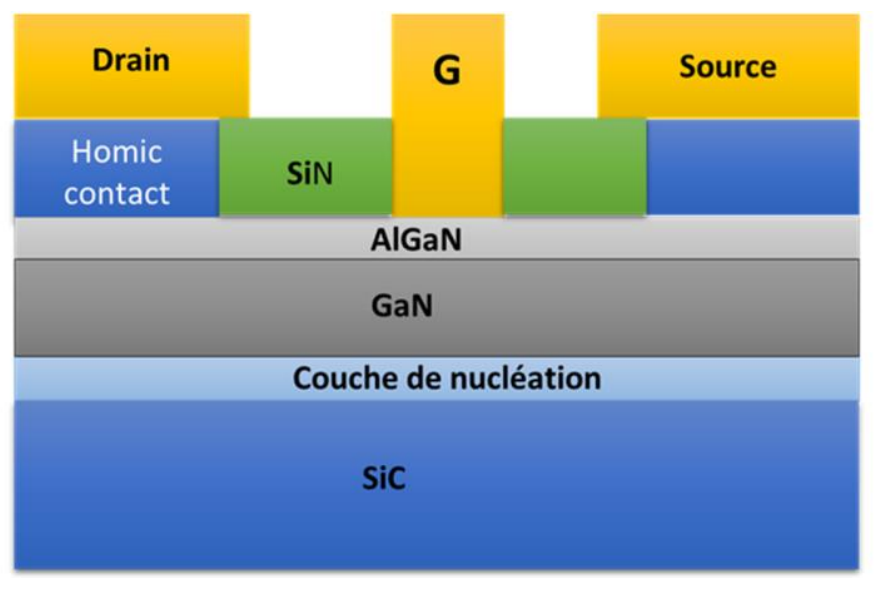

Figure 1. La structure du HEMT

\section{Modélisation électro-thermomécanique du HEMT}

La modélisation électrothermique permit d'étudier de comportement thermique du transistor à haute mobilité d'électron HEMT, lors de l'application d'une tension aux bornes du transistor, il dissipe une puissance sous forme de chaleur au niveau de la zone active à coté de drain à la sortie de grille (Wilson, n.d.). Cette puissance est en fonction de la tension appliquée aux bornes et le courant qui traverse ce transistor telle que [LEN 15]:

$$
P_{\text {diss }}=V_{d s} * I_{d s}
$$

où $V_{d s}$ et $I_{d s}$ representent respectivement la tension et le courant qui circule entre les deux bornes : le drain et la source. D'autre part, Le transfert thermique dans ce composant se fait principalement par conduction, car la convection et le rayonnement sont négligeables ils ne représentent que moins de $1,5 \%$ du transfert global [AUB 04]. D'où l'équation de la chaleur sera [ELH 15][AMA 19][AMA 21] :

$$
k \cdot \nabla^{2} T=\rho \cdot C_{P *} \frac{\partial T}{\partial t}+Q
$$


- Q la puissance dissipée in joules;

- K la conductivité thermique $\left(W \cdot m^{-1} \cdot K^{-1}\right) ; \rho$ density $\left(K g \cdot m^{-3}\right)$;

- $\quad C_{P}$ la capacité thermique massique $\left(J \cdot \mathrm{Kg}^{-1} \cdot \mathrm{K}^{-1}\right)$;

$\mathrm{T}$ the température $(\mathrm{K})$;

En plus des phénomènes thermiques, le fonctionnement du HEMT peut être accompagné des phénomènes mécaniques aussi. Par conséquence, une modélisation thermomécanique est nécessaire afin d'étudier l'influence du comportement thermique sur le comportement mécanique du HEMT. À cause du phénomène de l'auto échauffement lors de fonctionnement de HEMT sous des charges électriques, la température et sa distribution s'élève en influençant le comportement mécanique du composant. Cette influence peut se traduire sous forme des phénomènes et des dégradations mécaniques dans la structure du HEMT, telles que, les déplacements, les contraintes et les déformations.

Pour étudier ces phénomènes on fait appel à un modèle électro-thermomécanique, ce modèle permet de lier les paramètres thermiques comme la température aux paramètres mécaniques telles que les contraintes, déformations ou autres. Le couplage entre le modèle électrothermique et celui mécanique peut se faire grâce aux équations de la thermoélasticité, on peut définir les déformations et la densité d'entropie par les expressions suivantes [ELH 15, AMA 21] :

$$
\begin{gathered}
\{\varepsilon\}=[D]^{-1}\{\sigma\}+\{\alpha\} \Delta T \\
\{S\}=\{\alpha\}^{T}\{\sigma\}+\frac{\rho \cdot C_{P}}{T_{0}} \Delta T ; \Delta T=T-T_{\text {ref }}
\end{gathered}
$$

Où $\{\varepsilon\}$ est le vecteur de déformation totale, il est en fonction de vecteur des contraintes $\{\sigma\}$, le vecteur des coefficients de dilatation thermique, la différence de la température $\Delta T$ et l'inverse de la matrice de rigidité élastique $[D]$. Les deux couplages élémentaires: électrothermique et thermomécanique assurent le couplage global électro thermo mécanique.

Afin de construire le modèle électro thermo mécanique du HEMT, il est nécessaire de définir les propriétés thermiques et physiques des différents matériaux de la structure. Toutes les propriétés des matériaux nécessaires pour réaliser le modèle éléments finis sont classées dans le tableau 1 [LOR 13, MAU 13, SAU 07]. Quelques propriétés sont déjà intégrées dans la bibliothèque des matériaux de Comsol multiphysics.

\begin{tabular}{|c|c|c|c|c|c|c|}
\hline Matériaux & $\boldsymbol{\rho}[\mathrm{kg} / \mathrm{m3}]$ & $\mathbf{K T H}[\mathbf{W} / \mathbf{m} / \mathbf{K}]$ & $\mathbf{c p}[\mathbf{J} / \mathbf{k g} / \mathbf{K}]$ & $\mathbf{E}(\mathbf{G P a})$ & $\boldsymbol{\alpha}(\mathbf{K}-\mathbf{1})$ & $\mathbf{U}$ \\
\hline AlGaN & 5470 & $25 \cdot\left(\frac{293}{273+T}\right)^{-1,35}$ & 548 & 212,75 & $5.2425 \mathrm{e}-6$ & 0.319 \\
\hline GaN & 6100 & $161 \cdot\left(\frac{293}{273+T}\right)^{-1,45}$ & 490 & 181 & $8.6 \mathrm{e}-6$ & 0.352 \\
\hline SiC & 3220 & $416 \cdot\left(\frac{293}{273+T}\right)^{-1,5}$ & 690 & 181 & $4.3 \mathrm{e}-6$ & 0.45 \\
\hline $\begin{array}{c}\text { Interface } \\
\text { GaN/SiC }\end{array}$ & 6100 & $6,7 \cdot\left(\frac{293}{273+T}\right)^{-2,7}$ & 490 & 308 & $4.26 \mathrm{e}-6$ & 0.22 \\
\hline
\end{tabular}

Tableau 1. Propriétés thermiques des matériaux 


\section{Résultats des simulation numériques}

\subsection{Influence de la couche de nucléation sur le comportement électrothermique}

La puissance dissipée au niveau de la zone active du HEMT représente l'un des conditions du fonctionnement. On peut observer l'effet de cette puissance sur le comportement thermique du transistor, à l'aide du modèle éléments finis développé par le logiciel Comsol multiphysics, en gardant les autres paramètres constants, la température de référence était $25^{\circ} \mathrm{C}$. La figure montre la distribution de la température au sein de tout le HEMT. On peut remarquer aussi que la température est très importante au niveau de la couche de $\mathrm{AlGaN}$ à la sortie de la grille, autours de la couche de passivation et le long de la grille.

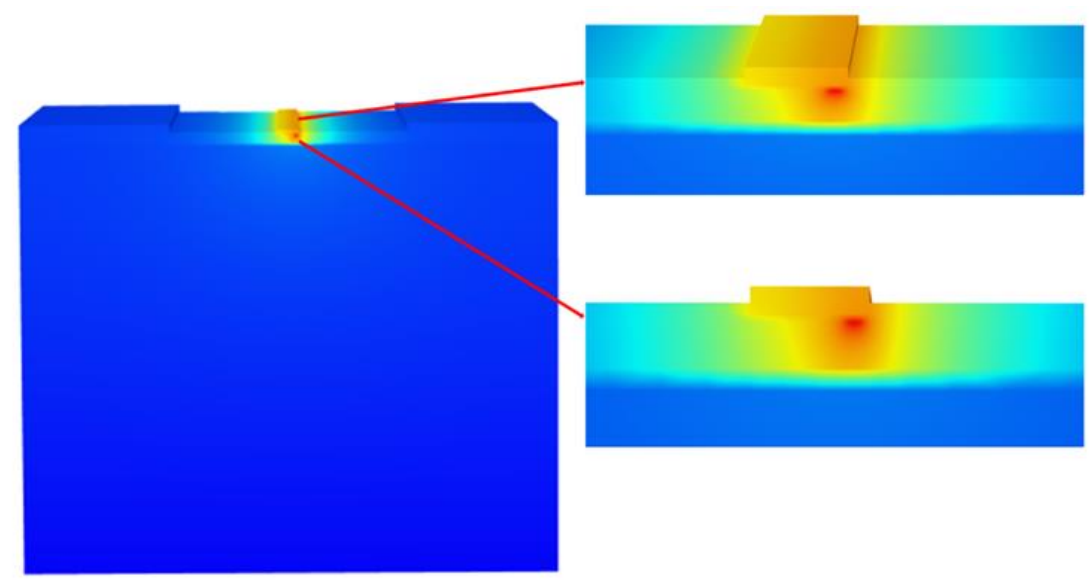

Figure 2. Distribution de la température dans la structure du HEMT

Dans le but d'identifier l'influence de paramètres géométriques de la structure du HEMT sur son comportement thermique et précisément sur la variation de la température du fonctionnement, on va investir dans le modèle éléments finis développé précédemment. Dans cette partie, on va s'intéresser à l'étude de la couche de nucléation et son influence sur le comportement thermique du HEMT. Comme on a déjà cité précédemment, la couche de nucléation joue un rôle très important dans la structure du HEMT. Nous avons réalisé des simulations numériques pour les différentes épaisseurs de la couche de nucléation en gardant la puissance dissipée et la température de référence constantes. Les résultats obtenus pour une puissance dissipée de $5 \mathrm{~W} / \mathrm{mm}$ et une température de référence de $25^{\circ} \mathrm{C}$ sont mentionnés dans la figure 3 .

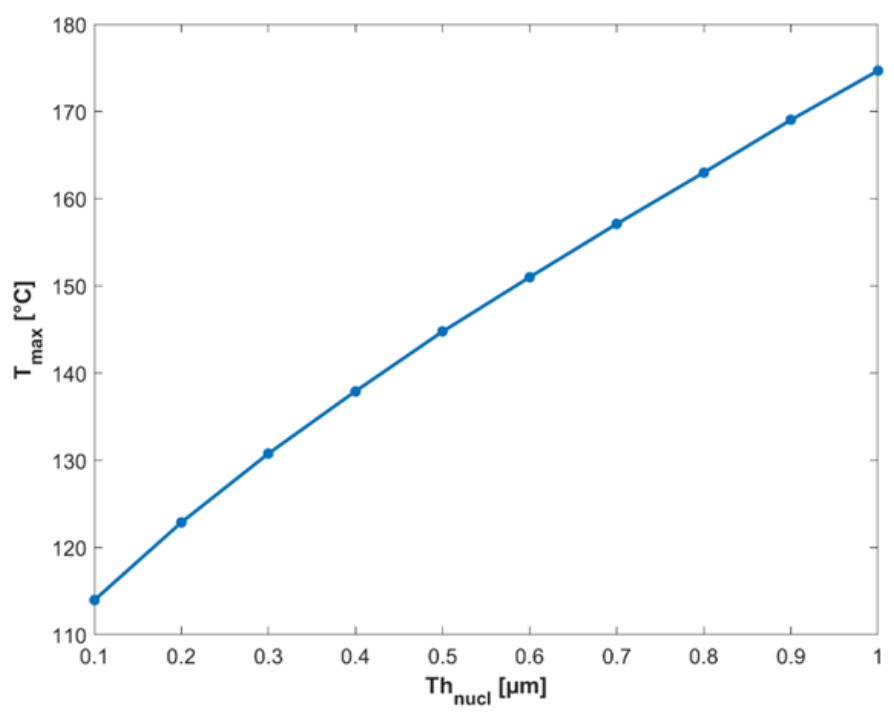

Figure 3. Evolution de la température en fonction de l'épaisseur de la couche de nucléation 


\subsection{Influence de la couche de nucléation sur le comportement thermomécanique du HEMT}

Comme les conditions du fonctionnement influencent le comportement thermique du HEMT, il influence également son comportement mécanique. La figure 4 montre la distribution des déplacements dans la structure du HEMT dans une puissance dissipée de $9 \mathrm{~W} / \mathrm{mm}$ et une température de référence de $150{ }^{\circ} \mathrm{C}$. Les déplacements sont très importants au niveau des trois électrodes du HEMT et surtout la grille, ce qui explique les défaillances qui se passe au niveau de ces électrodes.

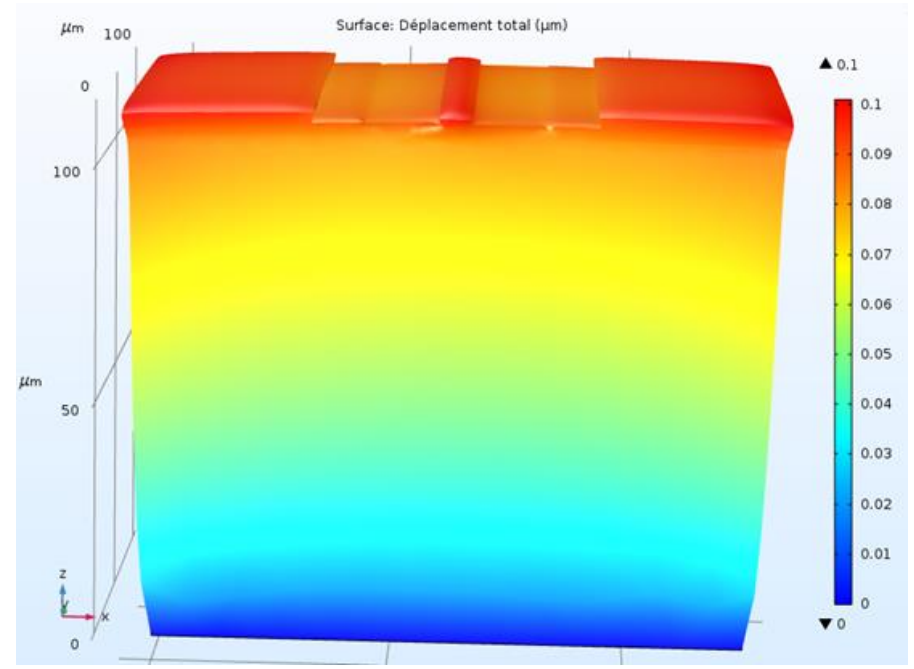

Figure 4. Distribution des déplacements dans la structure du HEMT

Dans la suite de cet article, on va étudier l'influence de la couche de nucléation sur le comportement thermomécanique de la structure du HEMT, grâce au modèle élément finis développé précédemment. Dans ce sens, nous allons appliquer les conditions du fonctionnement et les conditions aux limites en les gardant constantes. Par contre nous allons varier l'épaisseur de la couche de nucléation.

La figure 5 montre l'évolution des déplacements dans la structure de HEMT en fonction de l'épaisseur de la couche de nucléation. D'après cette figure on remarque que les déplacements augmentent en fonction l'épaisseur de la couche suivant une évolution non linéaire. Pour une variation de l'épaisseur entre 0.1 et $1 \mu \mathrm{m}$ on a une variation des déplacements entre 0.101 et 0.103 $\mu \mathrm{m}$.

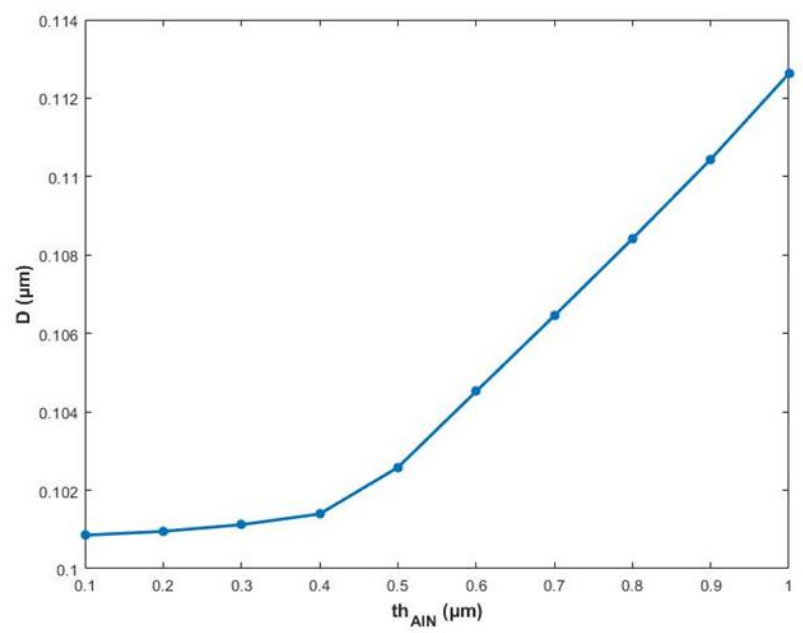

Figure 5. Evolution de la température en fonction de l'épaisseur de la couche de nucléation 
Pour une étude plus profonde, la figure 6 montre les résultats numériques des simulations de l'évolution des contraintes de von Mises dans la structure de HEMT en fonction de l'épaisseur de la couche de nucléation. On constate également que les contraintes équivalentes augmentent en fonction l'épaisseur de la couche suivant une évolution non linéaire. Dans notre cas, pour une variation de l'épaisseur entre 0.1 et $0.9 \mu \mathrm{m}$ on a une variation des contraintes entre 2.8 et $5 \mathrm{GPa}$.

D'apes tous ces résultats on constate que malgré que la couche de nucléation joue un rôle très important dans la structure de HEMT, il peut également influencer sa fiabilité si elle n'est pas bien maitrisée.

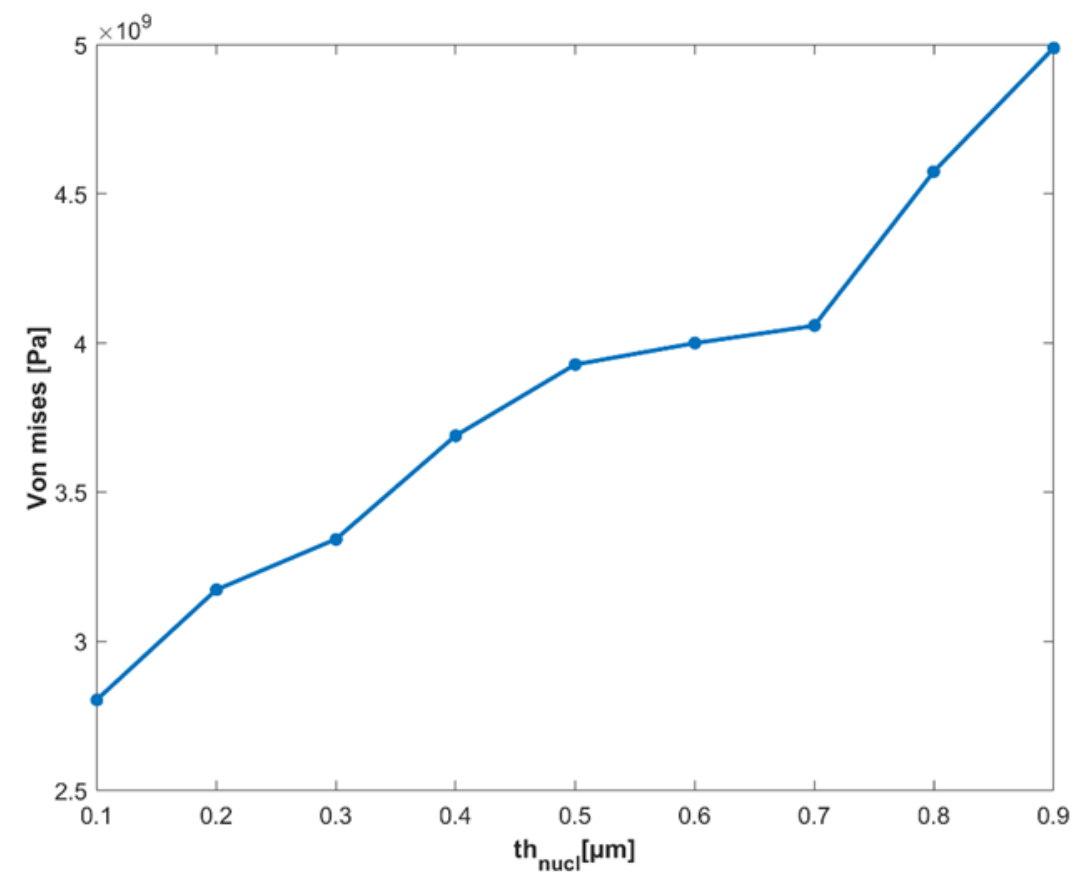

Figure 6. Evolution de la température en fonction de l'épaisseur de la couche de nucléation

\section{Conclusion}

Dans cet article, nous avons présenté la modélisation électro-thermomécanique du transistor à haute mobilité d'électron par la méthode des éléments finis, à l'aide de logiciel Comsol multiphysics. Ce modèle nous a permis de simuler le comportement thermomécanique du HEMT en fonction des conditions du fonctionnement. Il a permis également d'étudier l'influence de la couche de nucléation sur ce comportement. On a remarqué que malgré que l'épaisseur de la couche ne dépasse pas $1 \mu \mathrm{m}$, elle présente une grande influence sur le comportement thermique et mécanique du composent. Cette couche doit être prise en considération pour toute étude de développement ou d'amélioration du composant.

\section{Bibliographie}

[SAJ 20] S. S. Sajjadi Jaghargh and A. A. Orouji, "An AlGaN/GaN HEMT by a reversed pyramidal channel layer: Investigation and fundamental physics," Int. J. Numer. Model. Electron. Networks, Devices Fields, no. December 2019, pp. 13-18, 2020, doi: 10.1002/jnm.2719.

[AMA 21b] A. Amar, B. Radi, and A. El Hami, "Reliability based design optimization applied to the high electron mobility transistor (HEMT)," Microelectron. Reliab., vol. 124, no. July, p. 114299, 2021, doi: 10.1016/j.microrel.2021.114299.

[GAR 16] S. García, I. Ñiguez-De-La-Torre, J. Mateos, T. González, and S. Pérez, "Impact of substrate and thermal boundary resistance on the performance of $\mathrm{AlGaN} / \mathrm{GaN}$ HEMTs analyzed by means of electro-thermal Monte Carlo simulations," Semicond. Sci. Technol., vol. 31, no. 6, 2016, doi: 10.1088/0268-1242/31/6/065005. 
[JEO 18] D. Y. Jeon et al., "Effects of series resistance and interface properties on the operation of AlGaN/GaN high electron mobility transistors," Microelectron. Eng., vol. 199, pp. 40-44, 2018, doi: 10.1016/j.mee.2018.07.010.

[DON 19] Y. Dong, Z. Xie, D. Chen, H. Lu, R. Zhang, and Y. Zheng, "Effects of dissipative substrate on the performances of enhancement mode AlInN/GaN HEMTs," Int. J. Numer. Model. Electron. Networks, Devices Fields, vol. 32, no. 1, pp. 1-9, 2019, doi: 10.1002/jnm.2482.

[KAO 20] H. L. Kao, C. L. Cho, H. C. Chiu, H. Y. Wang, S. H. Chuang, and H. H. Hsu, "Mechanical tensile strain for $\mathrm{AlGaN} / \mathrm{GaN}$ metal-insulator-semiconductor high-electron-mobility transistors on a silicon-on-insulator substrate," J. Alloys Compd., vol. 820, p. 153178, 2020, doi: 10.1016/j.jallcom.2019.153178.

[LEN 15] L. Baczkowski, "Modélisation et Caractérisation Thermique de Transistors de Puissance Hyperfréquence GaN et Conséquences sur la Fiabilité de Modules Radars d'Émission/Réception en Bande X," Thèse de doctorat. Université de Lille, 2015.

[AMA 20b] A. Abdelhamid, R. Bouchaib, and E. H. Abdelkhalak, "Optimisation du comportement thermique du transistor à haute mobilité d'électron (HEMT) par la méthode KA-CMA-ES," Incert. fiabilité des systèmes multiphysiques, vol. 4, no. 2, pp. 1-11, 2020, doi: 10.21494/iste.op.2021.0600.

[AMA 20a] A. Abdelhamid, H. Hamid, R. Bouchaïb, and E. H. Abdelkhalak, "Optimisation thermique du transistor à haute mobilité d' électron ( HEMT ) par la méthode CMA -ES Thermal optimization of the high electron mobility transistor ( HEMT ) using the CMA-ES method," pp. 10-21, 2020.

[AMA 19] A. Amar, B. Radi, and A. El Hami, "La modélisation thermique de transistor à haute puissance de type HEMT," Incert. fiabilité des systèmes multiphysiques, vol. 3, no. 2019, pp. 1-7, 2019, doi: 10.21494/iste.op.2019.0431.

[COM 20] “COMSOL Multiphysics modeling software®.” https://www.comsol.fr/ (accessed Jan. 06, 2020).

[NIF 17] I. Nifa et al., "Characterization of 2DEG in AlGaN/GaN heterostructure by Hall effect," Microelectron. Eng., vol. 178, pp. 128-131, 2017, doi: 10.1016/j.mee.2017.05.009.

[AMA 21] A. Amar, B. Radi, and A. El Hami, "Electrothermal reliability of the high electron mobility transistor (Hemt)," Appl. Sci., vol. 11, no. 22, 2021, doi: 10.3390/app112210720.

[AUB 04] R. Aubry, "Etude des aspects électrothermiques de la fikière HEMT AlGaN/GaN pour application de puissance hyperfréquence," Thèse de doctorat. L'université des sciences et technologiques de Lille, 2004.

[ELH 15] A. El Hami and P. Pougnet, Embedded Mechatronic Systems 2 : Analysis of Failures, Modeling, Simulation and Optimization. ISTE éditions, 2015.

[LOR 13] Lorin E. Stevens, "Thermo-Piezo-Electro-Mechanical Simulation of AlGaN (Aluminum Gallium Nitride) / GaN (Gallium Nitride) High Electron Mobility Transistor,” Utah State University, 2013.

[MAU 13] M. A. Der Maur and A. Di Carlo, "AlGaN / GaN HEMT Degradation: An Electro-Thermo-Mechanical Simulation," IEEE Trans. Electron Devices, vol. 60, no. 10, pp. 3142-3148, 2013.

[SAR 07] A. Sarua et al., "Thermal boundary resistance between GaN and substrate in AlGaN/GaN electronic devices," IEEE Trans. Electron Devices, vol. 54, no. 12, pp. 3152-3158, Dec. 2007, doi: 10.1109/TED.2007.908874. 